CARVALHO ADF; SILVA GO; PEREIRA RB; PINHEIRO JB. 2015. Produtividade e tolerância à queima-das-folhas de diferentes genótipos de cenoura de verão. Horticultura Brasileira 33: 299-304. DOI: http://dx.doi.org/10.1590/S0102-053620150000300004

\title{
Produtividade e tolerância à queima-das-folhas de diferentes genótipos de cenoura de verão
}

\author{
Agnaldo DF Carvalho${ }^{1}$; Giovani O Silva² ${ }^{2}$ Ricardo B Pereira ${ }^{1}$; Jadir B Pinheiro ${ }^{1}$ \\ 'Embrapa Hortaliças, Brasília-DF, Brasil; agnaldo.carvalho@embrapa.br; ricardo-borges.pereira@embrapa.br; jadir.pinheiro@embrapa. \\ br; ${ }^{2}$ Embrapa Produtos e Mercado, Canoinha-SC, Brasil; giovani.olegario@embrapa.br
}

\begin{abstract}
RESUMO
A substituição de cultivares de cenoura de polinização livre por híbridos é uma realidade no mercado de sementes. Esse trabalho teve como objetivos avaliar o potencial produtivo de híbridos experimentais de cenoura, a tolerância à queima-das-folhas bem como a uniformidade de raízes, comparado a cultivares de polinização livre. Dezoito genótipos de cenoura $\{15$ híbridos experimentais, um híbrido comercial (Juliana) e duas cultivares de polinização livre (OPs) (Brasília e BRS Planalto)\} foram avaliados na safra 2011/12 em Brasília-DF em delineamento de blocos ao acaso com três repetições em parcelas de $2 \mathrm{~m}^{2}$. Aos 90 dias foi avaliada a incidência da queima-das-folhas e aos 100 dias foi realizada a mensuração dos caracteres componentes de produção, além da tomada ao acaso de 50 raízes por parcela para medidas individuais. Entre os genótipos avaliados houve diferença para todos os caracteres avaliados, existindo híbridos superiores ou inferiores às cultivares $O P S$. No contraste entre grupos de cultivares, não houve diferença entre híbridos e $O P S$ para produção total de raízes. No entanto, os híbridos apresentaram maior quantidade de raízes comerciais quando comparados às $O P S$. Em relação à uniformidade no comprimento de raízes, as cultivares OPs foram semelhantes aos híbridos, mas mais desuniformes em relação a diâmetro e peso individual. Os resultados demonstram a superioridade dos híbridos em relação às cultivares $O P s$ para produção comercial e uniformidade de raízes.
\end{abstract}

Palavras-chave: Daucus carota, Alternaria dauci, Cercospora carotae, rendimento, uniformidade.

\begin{abstract}
Productivity and tolerance to the leaf blight disease of hybrid and open-pollinated carrot cultivars

The replacement of open-pollinated carrot cultivars by hybrids is a reality in the seed market. We aimed to evaluate the productive potential of experimental carrot hybrids, tolerance to leaf blight as well as the uniformity of roots, compared to open-pollinated cultivars. Eighteen carrot genotypes, 15 experimental hybrids, one commercial hybrid (Juliana) and two open-pollinated cultivars (OPS) (Brasília and BRS Planalto) were evaluated in the 2011/12 crop in Brasília, Brazil, in randomized blocks design with three replications in plots of $2 \mathrm{~m}^{2}$. At 90 days the incidence of leaf blight was evaluated and at 100 days we measured production component characters, in addition to taking at random 50 roots per plot for individual measures. Difference was observed for all traits among the genotypes, hybrid being better or worse than $O P S$. For total root yield, no contrast was found between hybrids and OPs. However, hybrids showed higher amount of marketable roots compared to OPS. Regarding uniformity of root lenght, $O P S$ were similar to hybrids, but more irregular in relation to diameter and individual weight. The results demonstrate superiority of hybrids in relation to OPS for marketable production and uniformity of roots.
\end{abstract}

Keywords: Daucus carota, Alternaria dauci, Cercospora carotae, yield, uniformity.

\section{(Recebido para publicação em 5 de fevereiro de 2014; aceito em 13 de março de 2015) (Received on February 5, 2014; accepted on March 13, 2015)}

\begin{abstract}
A cenoura é a principal representante das apiáceas cultivadas mundialmente e umas das cinco principais espécies olerícolas no Brasil. Destaca-se entre as raízes tuberosas, pois, além de carboidratos, fibras e minerais, apresenta altos teores de carotenóides, os quais são convertidos em vitamina A no organismo (Olson, 1994; Packer et al., 1999). No Brasil são colhidos anualmente mais de 700 mil toneladas dessa hortaliça em aproximadamente 25 mil ha cultivados.
\end{abstract}

A cenoura é cultivada nas regiões Sul, Sudeste, Centro-Oeste e Nordeste brasileiro destacando as regiões de São Gotardo-MG, Marilândia do Sul-PR, Irecê-BA e Cristalina-GO (Anuário Brasileiro de Hortaliças, 2012).

No Brasil a produção de cenoura teve um grande impulso com o lançamento da cultivar Brasília de polinização livre (Open Pollination, OPs) em 1981. Antes disso, havia uma sazonalidade de produção o que fazia com que a oferta de cenoura fosse pequena durante a safra de verão e o preço aumentasse consideravelmente nesse período (Vieira et al., 1983). Seleções de genótipos de cenouras dentro da população da cultivar Brasília deram origem a várias cultivares. $\mathrm{O}$ resultado disso foi que o genótipo original se transformou em referência do grupo de cenouras de verão, dando origem, ao que se conhece hoje como 'grupo Brasília' ou cenouras de verão. 
A Embrapa, depois do lançamento da 'Brasília', lançou as cultivares Alvorada e mais recentemente BRS Planalto, cenouras de mesa, resultado de melhoramento genético de populações de 'Brasília' (Vieira et al., 2012). A cultivar BRS Planalto, lançada em 2009, que também é $O P$, apesar de elevado potencial produtivo, elevado nível de tolerância à queima-das-folhas e significativas melhorias na qualidade de raízes (Vieira et al., 2012), não é competidora dos híbridos modernos que apresentam ampla aceitação comercial. Assim, a Embrapa tem trabalhado no melhoramento genético visando obter cultivares de cenoura híbrida para plantios de verão.

Em países desenvolvidos, a utilização de sementes híbridas de cenoura chega a quase $100 \%$ da área plantada. Nesses países, desde 1960 os produtores vêm se beneficiando dessa tecnologia (Peterson \& Simon, 1985; Simon, 2000). No Brasil, há muitos anos, praticamente toda cenoura cultivada no inverno é híbrida. No entanto, no verão os primeiros híbridos surgiram no ano 2000 e logo substituiu em grande parte o cultivo de cenouras de polinização livre em regiões em que se emprega alta tecnologia de produção (Vieira et al., 2009).

A vantagem comparativa entre cultivares híbridas e $O P s$ fez com que o mercado de sementes de cenoura, aumentasse a demanda por cultivares híbridas, uma vez que esses genótipos possuem vantagens sobre as cultivares $O P S$ já que podem ser mais precoces, uniformes e com maior teto produtivo (Kutka, 2011). Embora possuam custo mais elevado, com a aquisição de sementes os produtores que adotam alta tecnologia preferem sementes híbridas, pois o ganho com produtividade e qualidade de raízes consegue saldar o gasto adicional com a aquisição das sementes (Pierro, 2003; Hasegawa, 2006).

A queima-das-folhas é uma das principais doenças foliares da cultura da cenoura (Pryor, et al., 2002). Estudos comparando correlação entre os sintomas do ataque da queima-das-folhas e produção são associados e negativos, ou seja, quanto maior a incidência de sintomas, maiores serão os reflexos no ren- dimento de raízes comerciais (Brito et al., 1997; Pereira et. al., 2012). Contudo, essa associação se reduz ou desaparece na medida em que os genótipos são mais tolerantes à queima-das-folhas, como demostrado por Pereira et al. (2012).

Diante do exposto, esse trabalho teve como objetivos avaliar o potencial produtivo de híbridos experimentais de cenoura, a tolerância à queima-das-folhas bem como a uniformidade de raízes, comparado a cultivares de polinização livre.

\section{MATERIAL E MÉTODOS}

O experimento foi instalado em Brasília-DF em novembro de 2011. Foram avaliados 18 genótipos de cenoura $\{15$ híbridos experimentais (CNPH-1 até CNPH-15) e três testemunhas \{Juliana (híbrido $\mathrm{F}_{1}$ ), BRS Planalto e Brasília (cultivares de polinização livre)\}. O delineamento experimental utilizado foi blocos ao acaso com três repetições. As parcelas foram constituídas de um canteiro com $2 \mathrm{~m}$ de comprimento por 1,0 $\mathrm{m}$ de largura, totalizando área de $2,0 \mathrm{~m}^{2}$. O sulco de plantio foi feito no sentido transversal ao canteiro em espaçamento duplo, sendo o espaçamento entre linhas simples de $10 \mathrm{~cm}$ e entre fileiras duplas de $20 \mathrm{~cm}$. O desbaste foi realizado aos 30 dias após o semeio deixando um espaço de $5 \mathrm{~cm}$ entre plantas, o que resultou numa população equivalente a 890 mil plantas/ha.

A adubação de plantio foi realizada com a formulação comercial N-P-K 0414-08 na dose de 1,2 t/ha. A adubação de cobertura foi realizada 35 dias após a semeadura com sulfato de amônio na dose de $400 \mathrm{~kg} / \mathrm{ha}$. O controle de plantas daninhas foi realizado três dias após a semeadura com a aplicação do herbicida Linuron, na dose de 900 mL/ ha do i.a. Na fase de desenvolvimento da cenoura o controle das plantas daninhas foi realizado com capina manual. A irrigação, quando necessária, foi realizada por aspersão com lâmina suficiente para manter o solo na capacidade de campo. Não foi aplicado agroquímico visando o controle de doenças fúngicas ou bacterianas.

A infecção por queima-das-folhas ocorreu naturalmente em campo. Os níveis de infecção foram considerados adequados, pois, parcelas de genótipos conhecidamente suscetíveis apresentaram alta severidade de ataque e infecção uniforme.

Aos 90 dias após a semeadura foi avaliada a severidade da queima-das-folhas (QDF), utilizando-se uma escala de notas de 1 a 5 para as parcelas $(1=$ mais que $90 \%$ de severidade, $2=50$ a $90 \%, 3=12,5$ a $50 \%, 4=3,8$ a $12,55 \%$ e $5=$ menor que $3,8 \%$ de severidade).

Aos 100 dias após a semeadura foi realizada a colheita, mensurando e transformando-se para $\mathrm{t} / \mathrm{ha}$ os caracteres: massa de raízes comerciais (MRC), massa de raízes totais (MRT) e massa de raízes refugo (MRR). Da classe comercial de raízes foram tomadas medidas individuais de 20 raízes, obtendo-se a massa média das raízes comerciais (MMR) em gramas, comprimento médio das raízes comerciais (CMR) e diâmetro médio das raízes comerciais (DMR), em centímetros.

De cada parcela experimental também foram tomadas ao acaso uma amostra de 50 raízes, as quais foram individualmente pesadas e medidas em comprimento e diâmetro, utilizando para essa última avaliação o terço médio superior, obtendo os caracteres massa de raízes individuais (MRI) em gramas, comprimento de raízes individuais (CRI) e diâmetro de raízes individuais (DRI) em centímetros.

Para os caracteres MRC, MRT, MRR, QDF, MMR, CMR e DMR os dados foram submetidos às pressuposições da análise de variância. A variável QDF foi transformada por $\sqrt{x+0,5}$ para atender às pressuposições. A análise de variância foi realizada utilizando o aplicativo computacional SAS v. 9.2 (SAS, 2008) e o teste de F utilizado para comparar as médias ajustadas entre grupos de cultivares. Para a comparação entre genótipos utilizou-se o teste de agrupamento de Scott-Knott com o software Genes v.2009.7.0 (Cruz, 2006).

Para a comparação da uniformidade entre os tipos de cultivares foram utilizados os caracteres MRI, CRI e DRI, por meio do aplicativo computacional SAS v. 9.2 (proc ttest). Por meio desta análise 
foram estimadas as médias populacionais, os limites superior e inferior, além do desvio e erro padrão. Foi estimado também o teste de $F$ para igualdade de variância (folded test) e teste de $t$ para comparação de médias entre tipos de cultivares, ajustando os graus de liberdade por Satterthwaite para variâncias diferentes entre grupos.

\section{RESULTADOS E DISCUSSÃO}

Houve diferenças significativas pelo teste de $\mathrm{F}$ entre híbridos e $O P s$ para MRR, QDF, MRC e CMR $(\mathrm{p}<0,01)$ (dados não apresentados). Em relação à produtividade total (MRT) não foram observadas diferenças entre híbridos e cultivares $O P S$.

Na Tabela 1 estão apresentados os resultados do teste de agrupamento de médias de Scott-Knott $(\mathrm{p}<0,05)$ entre genótipos e entre grupos de genótipos, o coeficiente de variação experimental (CV) e a média geral para cada caráter avaliado. Os CVs variaram de 6,46 a $34,15 \%$ para os caracteres diâmetro médio de raízes (DMR) e severidade da queima-das-folhas (QDF), respectivamente. Esses valores são considerados de boa precisão e estão próximos dos valores de coeficientes de variação em experimentos de campo com a cultura de cenoura (Luz, et al., 2000; Oliveira et al., 2005; Silva et al., 2011).
Entre genótipos observou-se que existem híbridos superiores ou inferiores às cultivares $O P S$ para todos os caracteres avaliados (Tabela 1). No entanto, de maneira geral, os híbridos se mostraram mais promissores, pois apresentaram alta produtividade de raízes comerciais (MRC), totais (MRT) e pouco refugo (MRR). Em relação à QDF os híbridos foram superiores às cultivares $O P S$.

A porcentagem de infecção observada para as populações OPs deve-se à maior suscetibilidade de 'Brasília' à QDF (Tabela 1), o que fez a média das populações aumentarem e serem significativamente diferentes dos híbridos. Não houve diferenças significativas

Tabela 1. Médias ajustadas de 18 genótipos de cenoura, híbridos e populações de polinização livre, avaliados no Distrito Federal, safra 2011/12 (adjusted means of 18 carrot genotypes, hybrids and open pollination cultivars, evaluated in Distrito Federal, 2011/12). Brasília, Embrapa Hortaliças, 2013.

\begin{tabular}{|c|c|c|c|c|c|c|c|c|c|c|c|c|c|c|c|}
\hline \multicolumn{2}{|l|}{ Genótipo } & \multicolumn{2}{|c|}{ MRR } & \multicolumn{2}{|l|}{ MRC } & \multicolumn{2}{|c|}{ MTR } & \multicolumn{2}{|c|}{ QDF } & \multicolumn{2}{|l|}{ MMR } & \multicolumn{2}{|l|}{ CMR } & \multicolumn{2}{|l|}{ DMR } \\
\hline CNPH-1 & $\mathrm{H}^{1}$ & 6,10 & $b^{2}$ & 23,74 & $\bar{b}$ & 29,85 & $\overline{\mathrm{c}}$ & 32,68 & $\bar{a}$ & 109,33 & $\bar{a}$ & 18,89 & $\bar{a}$ & 3,04 & $\bar{a}$ \\
\hline CNPH-2 & $\mathrm{H}$ & 7,80 & $\mathrm{~b}$ & 37,78 & $\mathrm{a}$ & 45,58 & $\mathrm{a}$ & 8,15 & $\mathrm{~b}$ & 118,83 & $\mathrm{a}$ & 18,56 & $\mathrm{a}$ & 3,05 & $\mathrm{a}$ \\
\hline CNPH-3 & $\mathrm{H}$ & 4,82 & $\mathrm{~b}$ & 37,05 & $\mathrm{a}$ & 41,87 & $\mathrm{a}$ & 10,14 & $\mathrm{~b}$ & 109,64 & $\mathrm{a}$ & 17,13 & $\mathrm{~b}$ & 3,09 & $\mathrm{a}$ \\
\hline CNPH-4 & $\mathrm{H}$ & 11,13 & $\mathrm{a}$ & 30,89 & $\mathrm{~b}$ & 42,02 & $\mathrm{a}$ & 10,14 & $\mathrm{~b}$ & 118,20 & $\mathrm{a}$ & 18,45 & $\mathrm{a}$ & 3,12 & $\mathrm{a}$ \\
\hline CNPH-5 & $\mathrm{H}$ & 12,19 & $\mathrm{a}$ & 30,23 & $\mathrm{~b}$ & 42,42 & $\mathrm{a}$ & 23,26 & $\mathrm{a}$ & 124,41 & $\mathrm{a}$ & 17,91 & $\mathrm{~b}$ & 3,20 & $\mathrm{a}$ \\
\hline CNPH-6 & $\mathrm{H}$ & 5,41 & $\mathrm{~b}$ & 36,42 & $\mathrm{a}$ & 41,83 & $\mathrm{a}$ & 7,03 & $\mathrm{~b}$ & 133,55 & $\mathrm{a}$ & 19,29 & $\mathrm{a}$ & 3,14 & $\mathrm{a}$ \\
\hline CNPH-7 & $\mathrm{H}$ & 8,34 & $\mathrm{~b}$ & 29,07 & $\mathrm{~b}$ & 37,41 & $\mathrm{~b}$ & 32,68 & $\mathrm{a}$ & 105,95 & $\mathrm{~b}$ & 17,54 & $\mathrm{~b}$ & 2,97 & $\mathrm{a}$ \\
\hline CNPH-8 & $\mathrm{H}$ & 7,78 & $\mathrm{~b}$ & 23,78 & $\mathrm{~b}$ & 31,56 & $\mathrm{c}$ & 15,31 & $\mathrm{~b}$ & 105,62 & $\mathrm{~b}$ & 18,50 & $\mathrm{a}$ & 2,80 & $\mathrm{~b}$ \\
\hline CNPH-9 & $\mathrm{H}$ & 6,70 & $\mathrm{~b}$ & 27,68 & $\mathrm{~b}$ & 34,38 & $\mathrm{~b}$ & 37,20 & $\mathrm{a}$ & 91,87 & $\mathrm{~b}$ & 17,06 & $\mathrm{~b}$ & 2,76 & $\mathrm{~b}$ \\
\hline CNPH-10 & $\mathrm{H}$ & 4,38 & $\mathrm{~b}$ & 32,34 & a & 36,71 & $\mathrm{~b}$ & 7,03 & $\mathrm{~b}$ & 124,30 & $\mathrm{a}$ & 19,05 & $\mathrm{a}$ & 3,13 & $\mathrm{a}$ \\
\hline CNPH-11 & $\mathrm{H}$ & 6,42 & $\mathrm{~b}$ & 37,90 & $\mathrm{a}$ & 44,32 & $\mathrm{a}$ & 13,79 & $\mathrm{~b}$ & 123,86 & $\mathrm{a}$ & 19,22 & $\mathrm{a}$ & 3,08 & $\mathrm{a}$ \\
\hline CNPH-12 & $\mathrm{H}$ & 6,44 & $\mathrm{~b}$ & 28,36 & $\mathrm{~b}$ & 34,80 & $\mathrm{~b}$ & 37,20 & $\mathrm{a}$ & 110,36 & $\mathrm{a}$ & 18,60 & $\mathrm{a}$ & 2,94 & $\mathrm{~b}$ \\
\hline CNPH-13 & $\mathrm{H}$ & 8,96 & $\mathrm{~b}$ & 26,64 & $\mathrm{~b}$ & 35,59 & $\mathrm{~b}$ & 13,79 & $\mathrm{~b}$ & 97,60 & $\mathrm{~b}$ & 17,76 & $\mathrm{~b}$ & 2,86 & $\mathrm{~b}$ \\
\hline CNPH-14 & $\mathrm{H}$ & 6,90 & $\mathrm{~b}$ & 27,59 & $\mathrm{~b}$ & 34,49 & $\mathrm{~b}$ & 32,68 & $\mathrm{a}$ & 96,69 & $\mathrm{~b}$ & 17,52 & $\mathrm{~b}$ & 2,81 & $\mathrm{~b}$ \\
\hline CNPH-15 & $\mathrm{H}$ & 6,00 & $\mathrm{~b}$ & 31,02 & $\mathrm{~b}$ & 37,02 & $\mathrm{~b}$ & 10,14 & $\mathrm{~b}$ & 99,51 & $\mathrm{~b}$ & 18,70 & $\mathrm{a}$ & 2,84 & $\mathrm{~b}$ \\
\hline Juliana & $\mathrm{H}$ & 13,57 & $\mathrm{a}$ & 7,59 & $\mathrm{~d}$ & 21,16 & $\mathrm{~d}$ & 50,62 & $\mathrm{a}$ & 75,83 & $\mathrm{c}$ & 13,21 & $\mathrm{c}$ & 2,85 & $\mathrm{~b}$ \\
\hline Brasília & $\mathrm{O}$ & 12,29 & $\mathrm{a}$ & 16,06 & $\mathrm{c}$ & 28,35 & $\mathrm{c}$ & 50,62 & $\mathrm{a}$ & 95,30 & $\mathrm{~b}$ & 14,39 & $\mathrm{c}$ & 3,07 & $\mathrm{a}$ \\
\hline Planalto & $\mathrm{O}$ & 10,88 & $\mathrm{a}$ & 25,81 & $\mathrm{~b}$ & 36,68 & $\mathrm{~b}$ & 31,25 & $\mathrm{a}$ & 109,73 & $\mathrm{a}$ & 17,43 & $\mathrm{~b}$ & 3,00 & $\mathrm{a}$ \\
\hline $\mathrm{H}$ & & 7,68 & $\mathrm{~b}$ & 29,26 & $\bar{a}$ & 36,94 & $\bar{a}$ & 19,38 & $\mathrm{a}$ & 109,10 & $\bar{a}$ & 17,96 & $\bar{a}$ & $2,98 \mathrm{a}$ & \\
\hline $\mathrm{O}$ & & 11,58 & $\mathrm{a}$ & 20,93 & $\mathrm{~b}$ & 32,52 & $\mathrm{a}$ & 40,36 & $\mathrm{~b}$ & 102,52 & $\mathrm{a}$ & 15,91 & $\mathrm{~b}$ & $3,04 \mathrm{a}$ & \\
\hline $\mathrm{CV}(\%)$ & & 16,52 & & 27,15 & & 17,55 & & 34,15 & & 15,40 & & 9,13 & & 6,46 & \\
\hline Méd. Aj. & & 8,12 & & 28,33 & & 36,45 & & 21,34 & & 108,36 & & 17,73 & & 2,99 & \\
\hline
\end{tabular}

${ }^{1} \mathrm{H}=$ híbrido (hybrid); $\mathrm{O}=$ cultivar de polinização livre (open pollination cultivars); ${ }^{2}$ médias seguidas de mesma letra na coluna não diferem estatisticamente pelo teste de $\mathrm{F}$ (means with the same letter in the column do not differ statistically by the F test). MRR= massa de raízes refugo $(\mathrm{t} / \mathrm{ha})\{$ mass of no marketable roots $(\mathrm{t} / \mathrm{ha})\} ; \mathrm{MRC}=$ massa de raízes comerciais $(\mathrm{t} / \mathrm{ha})\{$ mass of marketable roots $(\mathrm{t} / \mathrm{ha})\} ; \mathrm{MTR}=$ massa total de raízes ( $\mathrm{t} / \mathrm{ha}$ ) $\{$ total mass of roots ( $\mathrm{t} / \mathrm{ha})\} ; \mathrm{QDF}=$ severidade da queima-das-folhas (leaf blight severity); MMR= massa média de raízes comerciais $(\mathrm{g})$ \{average mass of marketable roots $(\mathrm{g})\} ; \mathrm{CMR}=$ comprimento médio de raízes comerciais $(\mathrm{cm})\{$ average length of marketable roots $(\mathrm{cm})\} ; \mathrm{DMR}=$ diâmetro médio de raízes comercias $(\mathrm{cm})$ average diameter of marketable roots $(\mathrm{cm})\}$. 
Tabela 2. Testes para igualdades de variância e comparação de médias entre genótipos híbridos e de polinização livre, para os caracteres medidos em raízes individuais, avaliadas no Distrito Federal, safra 2011/12 (variance equality test and means comparation among hybrids and open pollination genotypes, for characters measured in individual roots, evaluated in Distrito Federal, 2011/12). Brasília, Embrapa Hortaliças, 2013.

\begin{tabular}{|c|c|c|c|c|c|c|}
\hline \multicolumn{3}{|c|}{ Igualdade de variâncias } & \multicolumn{4}{|c|}{ Comparação entre médias de grupos } \\
\hline Variável & $\mathbf{F}_{c}$ & $\mathbf{P}>\mathbf{F}$ & Variâncias & GL & $\mathbf{t}_{c}$ & $\mathbf{P}>|\mathbf{t}|$ \\
\hline MMRi & 1,27 & 0,01 & diferente & 400 & 4,57 & $<, 0001$ \\
\hline CMRi & 1,09 & 0,35 & igual & 2671 & 7,48 & $<, 0001$ \\
\hline DMRi & 1,21 & 0,03 & diferente & 395 & 3,18 & 0,002 \\
\hline
\end{tabular}

$\mathrm{MMRi}=$ massa média de raízes individuais (mean mass of individual roots); $\mathrm{CMRi}=\mathrm{com}-$ primento médio de raízes individuais (mean lenght of individual roots); $\mathrm{DMRi}=$ diâmetro médio de raízes individuais (mean diameter of individual roots).

para MMR e DMR, apenas para CMR (17,96 cm dos híbridos contra 15,91 cm das $O P s$ ), sendo essa característica aproximadamente de $2 \mathrm{~cm}$ superior nos híbridos em relação às cultivares OPs. A não diferença entre híbridos e OPs para MMR e DMR pode ser explicada pelo fato das medidas serem realizadas em raízes comerciais, ou seja, com mesmo padrão comercial.

Entre os híbridos experimentais destacaram-se CNPH-2, CNPH-3, CNPH-6, CNPH-10 e CNPH-11. Foram esses genótipos que produziram maior quantidade de raízes comercializáveis, menor quantidade de refugo, menor incidência de queima-das-folhas (próximo a $10 \%$, maior massa média de raízes, maior comprimento médio de raízes (exceto $\mathrm{CNPH}-3$ ) e maior diâmetro médio de raízes. Esses híbridos foram superiores à testemunha Juliana e podem ser selecionados para testes de adaptabilidade em vários ambientes.

Em relação ao baixo desempenho do híbrido Juliana, vale ressaltar que os experimentos não receberam aplicação de fungicida, o que afetou consideravelmente o desempenho dessa cultivar, com reflexos negativos em todos os caracteres componentes de produção. Verificou-se ainda que a cultivar Brasília, mesmo com mesmo nível de severidade de queima-das-folhas $(50 \%)$, conseguiu superar 'Juliana' para a maioria dos caracteres avaliados. Esse fato demonstrou que 'Brasília' foi mais eficiente que 'Juliana' na produção e acúmulo de fotoassimilados em condições semelhantes de desfolha.

Para o teste de igualdade de variância para os caracteres massa de raízes individuais (MRI), comprimento de raízes individuais $(\mathrm{CRI})$ e diâmetro de raízes individuais (DRI) (Tabela 2), verificou-se que o caráter CRI apresentou variâncias não diferentes entre híbridos e OPs ( $p \geq 0,05)$. Em condições de igualdade de variâncias, utilizou-se para comparação entre médias o teste de agrupamento. Por esse teste verificou-se que a diferença entre as médias de híbridos e cultivares OPs é altamente significativa $(\mathrm{p}<0,01)$, sendo os híbridos superiores às cultivares OPs (Tabela 3 ). O comprimento médio para cada grupo encontra-se na Tabela 3. Nota-se que a média para CRI dos híbridos foi de $17,07 \mathrm{~cm}$, portanto superior à média de $15,17 \mathrm{~cm}$ das cultivares OPs. O comprimento superior dos híbridos em relação às OPs foi de $1,90 \mathrm{~cm}$, ou seja, proporcionalmente semelhante à diferença do caráter CMR (Tabela 1). Uma hipótese para explicar a variabilidade semelhante entre OPs e híbridos para essa característica é o tipo de crescimento que a cenoura apresenta nas diferentes fases do ciclo (Rubatzky et al., 1999); Crescimento primário (comprimento) durante os primeiros 45 dias após a semeadura e secundário (em diâmetro), dos 45 dias até próximo à colheita $( \pm 100$ dias para cenouras de verão). A ausência de competição por água, luz, nutrientes e plantas daninhas, nos primeiros 45 dias do ciclo, cria uma condição pouco adversa e assim favorável para que todas as plantas, tanto de OPs como de híbridos, tenham condições de crescimento até o máximo potencial genético de cada grupo de cultivar.

Tabela 3. Média para grupos, limites de confiança (LC) superior e inferior, desvio padrão e erro padrão para os grupos de genótipos híbridos e de polinização livre avaliadas no Distrito Federal na safra 2011/12 \{means for groups, superior and inferior confidence limits (LC), standard and error deviation of the groups of hybrid and open pollination genotypes evaluated in the Distrito Federal on the crop 2011/12\}. Brasília, Embrapa Hortaliças, 2013.

\begin{tabular}{llccccc}
\hline Características Cultivar & LC inferior & Média & LC superior & Desvio padrão & Erro padrão \\
\hline \multirow{3}{*}{ MMRi (g) } & Híbrida (H) & 102,42 & 104,32 & 106,21 & 47,16 & 0,97 \\
& Polinização livre (P) & 87,66 & 92,41 & 97,17 & 41,78 & 2,42 \\
& Dif (H-P) & 6,30 & 11,90 & 17,51 & 46,59 & 2,86 \\
\hline \multirow{3}{*}{ CMRi (cm) } & Híbrida (H) & 16,91 & 17,07 & 17,24 & 4,15 & 0,09 \\
& Polinização livre (P) & 14,72 & 15,17 & 15,63 & 3,97 & 0,23 \\
& Dif (H-P) & 1,40 & 1,90 & 2,40 & 4,13 & 0,25 \\
\hline \multirow{3}{*}{ DMRi (cm) } & Híbrida (H) & 2,89 & 2,91 & 2,93 & 0,56 & 0,01 \\
& Polinização livre (P) & 2,75 & 2,81 & 2,87 & 0,51 & 0,03 \\
& Dif (H-P) & 0,03 & 0,10 & 0,17 & 0,55 & 0,03 \\
\hline
\end{tabular}

$\mathrm{MMRi}=$ massa média de raízes individuais (mean mass of individual roots); $\mathrm{CMRi}=$ comprimento médio de raízes individuais (mean lenght of individual roots); $\mathrm{DMR}=$ diâmetro médio de raízes individuais (mean diameter of individual roots). 
Os caracteres MRI e DRI apresentaram variâncias diferentes entre híbridos e cultivares $O P S(\mathrm{p} \leq 0,05$, Tabela 2$)$. Nessa condição o teste entre médias de grupos é realizado com o ajuste dos graus de liberdade por Satterthwaite (variâncias diferentes, Tabela 2). Para MRI observou-se que a média dos híbridos foi de 104,32 $\mathrm{g}$ e a média para as OPs de 92,41g (Tabela 3). A diferença entre a massa média entre os grupos foi de 11,90 g, significativamente superior para os híbridos $(\mathrm{p}<0,01)$. Para DRI (Tabela 3), a média para os híbridos foi de 2,91 cm e 2,81 cm para as cultivares OPs. O valor da diferença entre grupos que foi de $0,10 \mathrm{~cm}$, também demonstra superioridade dos híbridos em relação às cultivares $O P S(\mathrm{p}<0,01)$.

Como já mencionado, após os 45 dias de semeadura a cenoura apresenta crescimento secundário, ou seja, em diâmetro. Nessa fase ocorre grande desenvolvimento na área foliar e as plantas começam a competir intensamente por luz, nutrientes, água e espaço (Watkinson, 1980; Li et al., 1996; Bernardi et al., 2004). A explicação para a maior variabilidade entre raízes de cultivares OPs para o caráter CRI provavelmente se deve à maior variabilidade genética entre indivíduos dentro de uma mesma população OP em relação a plantas de uma cultivar híbrida. Essa variabilidade parece não interferir na fase inicial que é o alongamento das raízes, mas interfere no crescimento em diâmetro, já que as plantas que estão mais vigorosas tendem a restringir o desenvolvimento das plantas menores e com isso não crescem o suficiente para atingir o tamanho das classes comercializáveis. Por outro lado, os híbridos possuem germinação mais uniforme e apresentam plântulas vigorosas (Simon et al., 2008). Após o crescimento primário, todas as plantas estão com o mesmo tamanho, com condições semelhantes de competição. Aliado a isso, os sistemas que empregam alta tecnologia proporcionam condições favoráveis ao desenvolvimento, pois nesses sistemas, não existem restrições quanto à irrigação, adubação, preparo de solo, máquinas de precisão para plantio e uso de agroquímicos. O resultado desse conjunto de fatores é uma condição altamente favorável para que a cultivar híbrida expresse seu potencial genético com grande quantidade de raízes com padrão comercial. Em condições de alta tecnologia as cultivares OPs também aumentam seu potencial produtivo, mas a capacidade de resposta dessas cultivares em relação aos híbridos é menor (Bandeira et al., 2013).

No caso da cenoura, a uniformidade de germinação e consequentemente estabelecimento das lavouras são fundamentais para o manejo da lavoura e para a colheita mecanizada. Nesse sentido, os resultados desse trabalho demonstram a superioridade dos híbridos de cenoura em rendimento de raízes comerciais por unidade de área cultivada e na uniformidade de raízes para massa e diâmetro. Além disso, como enfatizam Stein \& Nothnagel (1995), além de uniformidade de raízes, combinações híbridas podem reunir em um mesmo genótipo diferentes fontes de resistência a patógenos.

Os híbridos experimentais estudados foram superiores às cultivares $O P s$ e entre os híbridos os mais promissores foram CNPH-2, CNPH-3, CNPH-6, CNPH-10 e CNPH-11.

Entre os genótipos estudados os híbridos experimentais apresentaram maior uniformidade de raízes para massa e diâmetro.

\section{REFERÊNCIAS}

ANUÁRIO BRASILEIRO DE HORTALIÇAS. 2012. Brazilian Vegetable Yearbook. Santa Cruz do Sul: Gazeta. 88p.

BANDEIRA GLR; QUEIROZ SOP; ARAGÃO CA; COSTA ND; SANTOS CAF. 2013. Desempenho agronômico de cultivares de cebola sob diferentes manejos de irrigação no submédio São Francisco. Irriga 18:73- 84.

BERNARDI WF; FREITAS JA; SILVA VAR; TULMANN NETO A. 2004. Avaliação de espaçamentos de cenoura para os híbridos AF845 e AF750. Acta Scientiarum Agronomy 26: $125-130$.

BRITO CH; POZZA EA; JULIATTI FC; LUZ JMQ; PAES JMV. 1997. Resistência de cultivares de cenoura (Daucus carota) à queima das folhas durante o verão. Revista Ceres 44: 371-379.

CRUZ CD. 2006. Programa Genes: Estatística experimental e matrizes. Viçosa: Editora UFV. 285p.

HASEGAWA J. 2006. Estudo comprova rendimento de cenoura híbrida Horticeres.
Disponível em http://www.agrosoft.org.br/ agropag/20284.htm. Acessado em 25 de junho de 2013.

KUTKA F. 2011. Open-pollinated vs. hybrid maize cultivars. Sustainability 3: 1531-1554.

LI B; WATKINSON AR; HARA T. 1996. Dynamics of competition in populations of carrot (Daucus carota). Annals of Botany 78: 203-14.

LUZ JMQ; SILVA JÚNIOR JA; TEIXEIRA MSSC; SILVA MAD; SEVERINO GM; MELO B. 2000. Desempenho de cultivares de cenoura no verão e outono-inverno em Uberlândia-MG. Horticultura Brasileira 27: 096-099.

OLIVEIRA CD; BRAZ LT; BANZATTO DA. 2005. Adaptabilidade e estabilidade fenotípica de genótipos de cenoura. Horticultura Brasileira 23: 743-748.

OLSON JA. 1994. Absorption, transport, and metabolism of carotenoids in humans. Pure Applied Chemistry. 66: 1011-1016.

PACKER L; HIRAMATSU M; YOSHIKAWA T. 1999. Antioxidant food supplements in human health. Academic Press: Orlando. 511p.

PEREIRA RB, CARVALHO ADF, PINHEIRO JB, SILVA GO, VIEIRA JV. 2012. Resistência de populações de cenoura à queima-das-folhas com diferentes níveis de germoplasma tropical. Horticultura Brasileira 30: 489-493.

PETERSON CE; SIMON PW. 1985. Carrot breeding. In: BASSET MJ. (ed). Breeding Vegetable Crops. Westport: AVI 9: p.321-356.

PIERRO AC. 2003. Horticeres apresenta Juliana, primeira cenoura híbrida nacional de verão. Disponível em: http://www.paginarural.com. br/artigo/713/horticeres-apresenta-julianaprimeira-cenoura-hibrida-nacional-de-verao. Acessado em 26 de junho de 2013.

PRYOR BM; STRANDBERG JO; DAVIS RM; NUNEZ JJ; GILBERTSON RL. 2002. Survival and persistence of Alternaria dauci in carrot cropping systems. Plant Disease. 86: 1115-1122.

RUBATZKY VE; QUIROS CF; SIMON PW. 1999. Carrots and related vegetable Umbelliferae. Crop production science in horticulture 10: 294.

SAS INSTITUTE Inc. 2008. SAS/STAT ®9.2: User's guide: statistics version 9.2. North Carolina: Cary.

SILVA GO; CARVALHO ADF; VIEIRA JV; BENIN G. 2011. Verificação da adaptabilidade e estabilidade de populações de cenoura pelos métodos AMMI, GGE biplot e REML/BLUP. Bragantia 70: 494-501.

SIMON PW. 2000. Domestication, historical development, and modern breeding of carrot. Plant breeding reviews 19: 157-190.

SIMON PW; FREEMAN RE; VIEIRA JV; BOITEUX LS; BRIARD M; NOTHNAGEL T; MICHALIK B; KWON YS. 2008. Carrot. In: PROHENS J; NUEZ F. (eds). Handbook of Plant Breeding: Vegetables II: Fabaceae, Liliaceae, Solanaceae, and Umbelliferae. New York: Springer. p.327-357.

STEIN M; NOTHNAGEL TH. 1995. Some remarks on carrot breeding (Daucus carota sativus). Plant Breeding 114: 1-11. 
VIEIRA JV; DELLA VECCHIA PT; IKUTA H. 1983. Cenoura Brasília. Horticultura Brasileira 1: 42

VIEIRA JV; SILVA GO; BOITEUX LS; SIMON PW. 2009. Divergência genética entre acessos de cenoura pertencentes a grupos varietais distintos utilizando caracteres morfológicos. Horticultura Brasileira 27: 473-477.

VIEIRA JV; SILVA GO; CHARCHAR JM; FONSECAMEN; SILVAJBC; NASCIMENTO WM; BOITEUX LS; PINHEIRO JB; REIS A; RESENDE FV; CARVALHO ADF. 2012. BRS
Planalto: cultivar de cenoura de polinização aberta para cultivo de verão. Horticultura Brasileira 30: 359-363.

WATKINSON AR. 1980. Density-dependence in single-species populations of plants. Journal of Theoretical Biology 83: 345-357. 